\title{
SYNTHESIS OF NEW [1,5]-BENZODIAZEPINE DERIVATIVES CATALYZED BY VARIOUS ALIPHATIC ACIDS
}

\author{
Kamal M. El-Gaml \\ Organic chemistry department, Al-Azher University, Naser city, Cairo, 11884, Egypt
}

\begin{abstract}
:
A series of some new[1,5]benzodiazepines has been synthesized using condensation reaction of $o$-phenelendiamine with different newly synthesized quinolinyl ketones and effect of different aliphatic acids used as a catalyst was studied. Among the catalytic screened aliphatic acid, chloroacetic acid are a versatile catalyst that modify the yield (60-95\%) in short reaction time.
\end{abstract}

Keywords:, 1,5 - benzodiazepine , $o$-phenelendiamine , quinolinyl ketones, aliphatic acids .

\section{INTRODUCTION}

Benzodiazepines are important groups of heterocycle because they belong to class of pharmacologically pre-eminent, among this important class [1,5]-benzodiazepines which have extensively used as anti-convulsant, anti-anxiety, analgesic, sedative, anti-depressant, hypnotic (Randall, 1974). Anti-inflammatory (Roma, et al., 1991), antiviral (Di Braccio, et al., 2001), anti-microbial (Kumar and Joshi, 2007), and anti-tumor (Kamal, et al., 2008). The wide range of applications of benzodiazepines has attracted many researchers and various methods are known for their synthesis under different conditions. Among these methods condensation of $o$-phenelendiamine and ketones in the presence of a variety of catalysts like $p$-toluenesulfonic acid (Pasha and Jayashankara, 2006), silica sulfuric acid (Ahmad and Ali, 2007), p-nitrobenzoic acid (Varala, et al., 2008), sodium tetrachloroaurate dehydrate (Shi, et al., 2010), $\mathrm{AlCl}_{3}$ (More, et al., 2011) polyethylene glycol (Konda, et al., 2011). Unfortunately these methods involve many limitations like tedious work up procedure, producing undesired washes, applications of expensive catalyst and reagents, long reaction times, unsatisfactory yield, high purification cast.In last few years, there has been growing interest in the use of organic catalyst that can proceed and accomplish the reaction under aerobic atmosphere, other notable advantages are, commercially less expensive (Scheme 1) 

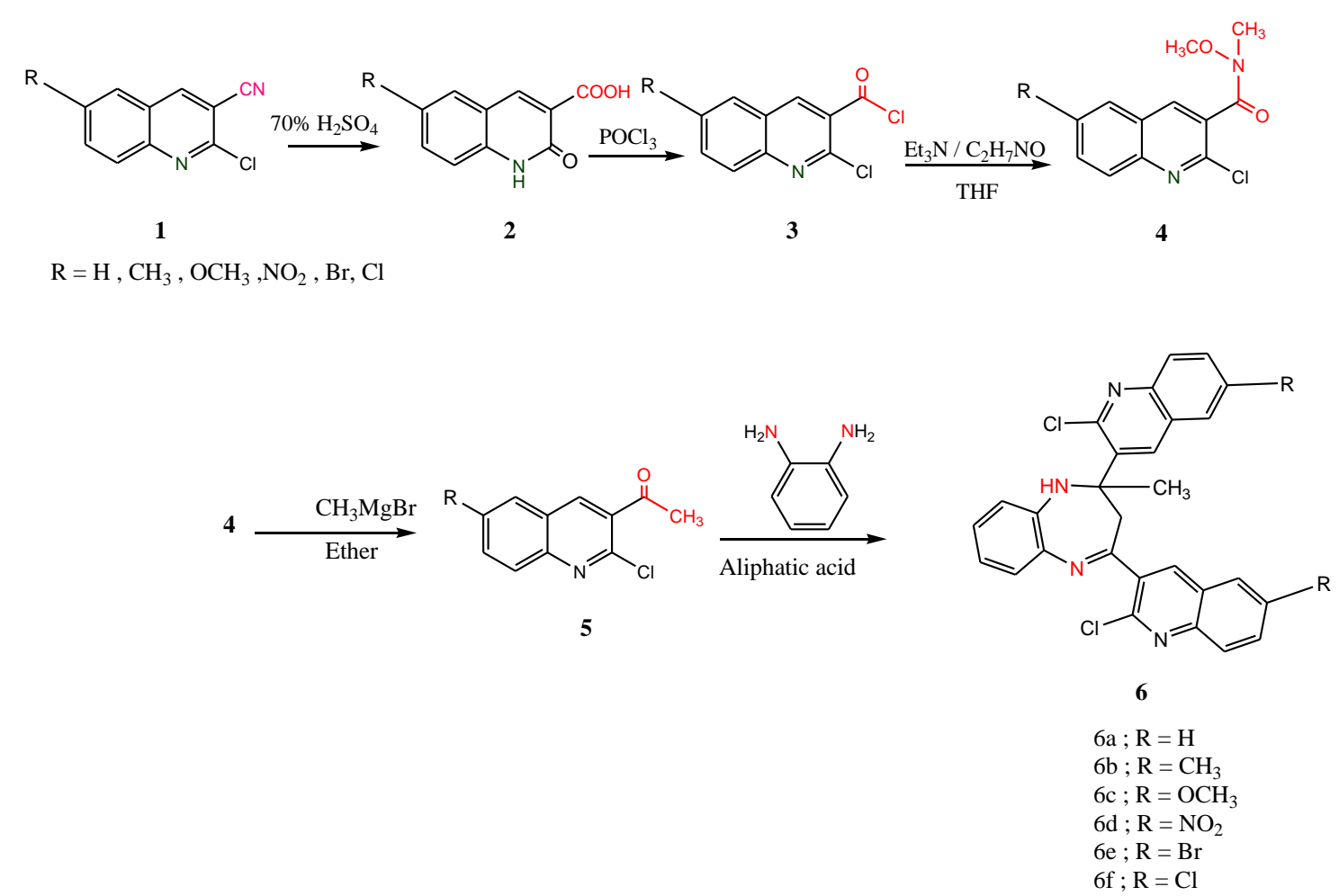

(Scheme1)

\section{EXPERIMENTAL}

\subsection{General}

1. All melting points were carried out on Gallenkamp melting point apparatus and are uncorrected. Microanalysis were carried out on Brucker-Vector -22-FT-IR spectrophotometer using the potassium bromide disc. ${ }^{1} \mathrm{HNMR}$ spectra were performed either on a Jeol ECA (500 MHz) or Gemini 300BB (300 MHz) spectrometer, using TMS as internal standard and DMSO-d6 as solvent; the chemical shifts are reported in ppm $(\delta)$ and coupling constant $(\mathrm{J})$ values are given in Hertz $(\mathrm{Hz})$. Signal multiplicities are represented by $\mathrm{s}$ (singlet), $\mathrm{d}$ (doublet), $\mathrm{t}$ (triplet), $\mathrm{q}$ (quadruplet), and $\mathrm{m}$ (multiplet). The impact ionization (IE) mass spectra were recorded on $\mathrm{AZH}-\mathrm{Ph}-\mathrm{AR}-\mathrm{XO}_{2}$ at 70 ev. Elemental analysis were performed on a $\mathrm{CHN}$ analyzer. All analyses were performed at the micro analytical center unit of cairo university ,cairo, Egypt. Acid Hydrolysis of $\mathbf{1}$ with $\mathrm{H}_{2} \mathrm{SO}_{4}$ (70\%) in ethanol gave substituted 2-oxo-2,3-dihydroquinoline-3-carboxylic acids (2) in good yield, when these compound were heated in boiling phosphoryl chloride it gave substituted 2-chloroquinoline-3-carbonyl chloride (3) . Subsequently, 3 were reacted with $\mathrm{N}, \mathrm{O}$-dimethyl hydroxylamine in the presence of triethylamine and THF, to give 2-chloro$N$-methoxy- $N$-methylquinoline-3-carboxamide derivatives (4), this on further reaction with methyl magnesium bromide gave 2-chloro-3-acetylquinoline derivatives (5), the structure of compounds 5 was confirmed on the basis of analytical and spectral data. The started compounds 1-3 were synthesized according to reported (Wright 1985; Neelima, 1986; Cyrous, 2006; Monir, 2004; Kamal, 2012) methods, in addition compounds 4 , 5, (6 ae) and newly prepared compound of were synthesized according to reported procedures 
(Kamal, 2012). The synthesis of the designed compounds was achieved following the routes depicted in Scheme 1.

3.1.1General procedure for synthesis of acid catalyzed newly substituted[1,5]benzodiazepines 6 a-f

A mixture of $o$-phenelendiamine (1mmole ), appropriate aliphatic acid (10 mol or $0.1 \mathrm{mmole})$ and various quinolinly ketones $(2.25 \mathrm{mmole})$ were refluxed on water bath for 5$6 \mathrm{hrs}$. After completion of the reaction monitored by TLC using chloroform and methanol (9.5:0.5ml) as eluent, the reaction mixture was poured into crashed ice and basified with ammonia solution, if required. The precipitated solid was separated, washed thoroughly with water and dried.

\section{2,4-bis(2-chloroquinolin-3-yl)-2-methyl-2,3 -dihydro-1H-benzo[b][ 1,5$]$ - diazepine (} 6a)' (Kamal, 2012)

Yield $85 \%$, m.p. $142-143{ }^{\circ} \mathrm{C} .\left(\mathrm{KBr}, \mathrm{cm}^{-1}\right): 3435,3110 .{ }^{1} \mathrm{HNMR}\left(\mathrm{DMSO}, \mathrm{D}_{2} \mathrm{O}\right): \delta$ $1.21\left(\mathrm{~s}, 3 \mathrm{H}, \mathrm{CH}_{3}\right.$ at $\left.\mathrm{C} 2\right), 2.20(\mathrm{~d}, 1 \mathrm{H}, \mathrm{J}=4.50 \mathrm{~Hz}), 3.10(\mathrm{~d}, 1 \mathrm{H}, \mathrm{J}=4.50 \mathrm{~Hz}), 4.24(\mathrm{~s}, 1 \mathrm{H}, \mathrm{NH}$ $\mathrm{D}_{2} \mathrm{O}$ exchangeable) 6.18-6.21(t, $2 \mathrm{H},-\mathrm{H}_{4}, \mathrm{H}_{5}$ of phenyl), 6.25-6.27(d, $2 \mathrm{H},-\mathrm{H}_{3}, \mathrm{H}_{6}$ of phenyl), 6.33-6.35(t, $2 \mathrm{H}, \mathrm{H}_{6}$ of quinoline), $6.97\left(\mathrm{~d}, 2 \mathrm{H}, \mathrm{H}_{5}\right.$ of quinoline $), 7.20-7.22\left(\mathrm{t}, 2 \mathrm{H}, \mathrm{H}_{7^{-}}\right.$ quinolin), 7.27-7.29(d, $2 \mathrm{H}, \mathrm{H}_{8}$ of quinoline), $8.15\left(\mathrm{~s}, 2 \mathrm{H}, \mathrm{H}_{4}\right.$ of quinoline); ${ }^{13} \mathrm{CNMR}$ (DMSO-d6) $\delta$ : 28.3, $\left(\mathrm{CH}_{3}\right), 42.3\left(\mathrm{CH}_{2}\right.$-benzodiazepine), 64.1 $(\mathrm{C}=\mathrm{N}), 114.3,118.5,123.2$, 124.3, 124.3, 126.5, 126.7, 127.1, 127.5, 127.2, 127.4, 127.9, 128.6, 129.8, 130.8, 131.3, 134.8, 137.3, 145.3, 145.9, 149.7, 153.6, 154.4, 162.7 . Anal. Calc. For $\mathrm{C}_{28} \mathrm{H}_{20} \mathrm{Cl}_{2} \mathrm{~N}_{4}$ : C, 69.57; H, 4.17; N, 11.59. Found. C, 69.28; H, 4.36; N, 11.63 .

\section{2,4-bis(2-chloro-6-methylquinolin-3-yl) $\quad$-2-methyl $\quad-2,3$-dihydro-1H-benzo[b][ 1,5$]$ - diazepine ( 6b) (Kamal, 2012)}

Yield $95 \%$, m.p. $138-139{ }^{\circ} \mathrm{C} .\left(\mathrm{KBr}, \mathrm{cm}^{-1}\right): 3432,3095,2841 .{ }^{1} \mathrm{HNMR}\left(\mathrm{DMSO}, \mathrm{D}_{2} \mathrm{O}\right), \delta$ : 1.12(s,3H, $\mathrm{CH}_{3}$-diazepine), $2.35(\mathrm{~d}, 1 \mathrm{H}, \mathrm{J}=3.65 \mathrm{~Hz}), 3.08(\mathrm{~d}, 1 \mathrm{H}, \mathrm{J}=3.65 \mathrm{~Hz}), 2.37(\mathrm{~s}, 6 \mathrm{H}-$ $\left.2\left(\mathrm{CH}_{3}\right)\right), 4.30\left(\mathrm{~s}, 1 \mathrm{H}, \mathrm{NH}, \mathrm{D}_{2} \mathrm{O}\right.$ exchangeable $)$. 6.16-6.21(t, $2 \mathrm{H},-\mathrm{H}_{4}, \mathrm{H}_{5}$ of phenyl), 6.266.28(d, $2 \mathrm{H},-\mathrm{H}_{3}, \mathrm{H}_{6}$ of phenyl), 6.85(s, $2 \mathrm{H}, \mathrm{H}_{5}$ of quinoline), 7.22-7.26(d, 2H, $\mathrm{H}_{7}$-quinolin), 7.30-7.32(d, $2 \mathrm{H}, \mathrm{H}_{8}$ of quinoline), $8.12\left(\mathrm{~s}, 2 \mathrm{H}-\mathrm{H}_{4}\right.$ of quinoline), Anal. Calc. For $\mathrm{C}_{30} \mathrm{H}_{24} \mathrm{Cl}_{2} \mathrm{~N}_{4}$ : C, 70.45; H, 4.73; N, 10.95. Found, C, 70.80; H, 4.50; N, 10.59 .

\section{2,4-bis(2-chloro-6-methoxyquinolin-3-yl) -2 -methyl $\quad-2,3$-dihydro-1H-benzo[b][ 1,5$]-$} diazepine ( 6c) (Kamal, 2012)

Yield $92 \%$, m.p. $121-122{ }^{\circ} \mathrm{C}$. IR (KBr, cm $\left.{ }^{-1}\right): 3430,3120,2835 .{ }^{1} \mathrm{HNMR}\left(\mathrm{DMSO}, \mathrm{D}_{2} \mathrm{O}\right), \delta$ : $1.10\left(\mathrm{~s}, 3 \mathrm{H}, \mathrm{CH}_{3}\right.$-diazepine), $2.21(\mathrm{~d}, 1 \mathrm{H}, \mathrm{J}=4.4 \mathrm{~Hz}), 3.09(\mathrm{~d}, 1 \mathrm{H}, \mathrm{J}=4.4 \mathrm{~Hz}), 3.60(\mathrm{~s}, 6 \mathrm{H}-$ 2(O-CH3), 3.94 (s, 1H, NH , $\mathrm{D}_{2} \mathrm{O}$ exchangeable). 6.22-6.26(t, $2 \mathrm{H}, \mathrm{H}_{4}, \mathrm{H}_{5}$ of phenyl), 6.306.32(d, $2 \mathrm{H}, \mathrm{H}_{3}, \mathrm{H}_{6}$ of phenyl), 6.88(s, 2H, $\mathrm{H}_{5}$ of quinoline), 7.23-7.25(d, 2H, $\mathrm{H}_{7}$-quinolin), 7.27-7.29(d, $2 \mathrm{H}, \mathrm{H}_{8}$ of quinoline), 8.11(s, $2 \mathrm{H}, \mathrm{H}_{4}$ of quinoline), Mass: $\mathrm{m} / \mathrm{z} 543.44$ (15.6, $\left.\mathrm{M}^{+}\right), 545\left(5.2, \mathrm{M}^{+2}\right)$. Anal. Calc. For $\mathrm{C}_{30} \mathrm{H}_{24} \mathrm{Cl}_{2} \mathrm{~N}_{4} \mathrm{O}_{2}: \mathrm{C}, 66.30 ; \mathrm{H}, 4.45 ; \mathrm{N}, 10.31$. Found. C, 66.13; H, 4.48; N, 10.15

\section{2,4-bis(2-chloro-6-nitroquinolin-3-yl) $\quad$-2-methyl $\quad-2,3 \quad$-dihydro-1H-benzo[b][ $\quad 1,5]-$ diazepine ( 6d) (Kamal, 2012)}

Yield $92 \%$, m.p. $150-152{ }^{\circ} \mathrm{C}$. IR $\left(\mathrm{KBr}, \mathrm{cm}^{-1}\right): 3405,3130,2840 .{ }^{1} \mathrm{HNMR}\left(\mathrm{DMSO}, \mathrm{D}_{2} \mathrm{O}\right)$, $\delta: 1.13\left(\mathrm{~s}, 3 \mathrm{H}, \mathrm{CH}_{3}\right.$-diazepine), $2.23(\mathrm{~d}, 1 \mathrm{H}, \mathrm{J}=4.75 \mathrm{~Hz}), 3.18(\mathrm{~d}, 1 \mathrm{H}, \mathrm{J}=4.75 \mathrm{~Hz}), 4.62(\mathrm{~s}$, $1 \mathrm{H}, \mathrm{NH}, \mathrm{D}_{2} \mathrm{O}$ exchangeable). $\quad 6.20-6.22\left(\mathrm{t}, 2 \mathrm{H}, \mathrm{H}_{4}, \mathrm{H}_{5}\right.$ of phenyl), 6.35-6.37(d, $2 \mathrm{H}, \mathrm{H}_{3}$, $\mathrm{H}_{6}$ of phenyl), 7.39-7.41(d, $2 \mathrm{H}, \mathrm{H} 8$-quinolin), 8.10(s, $2 \mathrm{H}, \mathrm{H}_{4}$ of quinoline), 8.48-8.50(d, $2 \mathrm{H}, \mathrm{H}_{7}$ of quinoline), 8.65 (s, $2 \mathrm{H}, \mathrm{H}_{5}$ of quinoline), Anal. Calc. For $\mathrm{C}_{28} \mathrm{H}_{18} \mathrm{Cl}_{2} \mathrm{~N}_{6} \mathrm{O}_{4}$ : C, 58.65; H, 3.16; N, 14.66. Found, C, 58.77; H, 2.97; N, 14.91 . 
2,4-bis(2-chloro-6-bromoquinolin-3-yl) $\quad$-2-methyl $\quad-2,3 \quad$-dihydro-1H-benzo[b][ 1,5$]-$ diazepine ( $6 \mathrm{e})^{16}$

Yield $92 \%$, m.p. $150-152{ }^{\circ} \mathrm{C}$. IR $\left(\mathrm{KBr}, \mathrm{cm}^{-1}\right): 3380$, 3097, 2815, . Mass: m/z 641.18 (9.14, $\left.\mathrm{M}^{+}\right), 643\left(3.41, \mathrm{M}^{+2}\right)$. Anal. Calc. For $\mathrm{C}_{28} \mathrm{H}_{18} \mathrm{Br}_{2} \mathrm{Cl}_{2} \mathrm{~N}_{4}$ : C, 52.45; H, 2.83; N, 8.74. Found, C, $52.13 ; \mathrm{H}, 3.15 ; \mathrm{N}, 8.80$.

\section{2,4-bis(2-chloro-6-bromoquinolin-3-yl) $\quad$-2-methyl $\quad-2,3 \quad$-dihydro-1H-benzo[b][ 1,5$]$ - diazepine ( 6f)}

Yield $92 \%$, m.p. $145-146{ }^{\circ} \mathrm{C}$ : : IR (KBr, cm $\left.{ }^{-1}\right)$ : 3452, 3105, 2910. ${ }^{1} \mathrm{HNMR}\left(\mathrm{DMSO}, \mathrm{D}_{2} \mathrm{O}\right)$, $\delta 1.12\left(\mathrm{~s}, 3 \mathrm{H}, \mathrm{CH}_{3}, \mathrm{C}\right.$-2-diazepine), $2.18(\mathrm{~d}, 1 \mathrm{H}, \mathrm{J}=4.55 \mathrm{~Hz}), 3.09(\mathrm{~d}, 1 \mathrm{H}, \mathrm{J}=4.55 \mathrm{~Hz}), 4.45$ (s, $1 \mathrm{H}, \mathrm{NH}, \mathrm{D}_{2} \mathrm{O}$ exchangeable), 6.29-6.31(t, 2H, $\mathrm{H}_{4}, \mathrm{H}_{5}$ of phenyl), 6.34-6.36(d, 2H, $\mathrm{H}_{3}, \mathrm{H}_{6}$ of phenyl), $7.33\left(\mathrm{~d}, 2 \mathrm{H}, \mathrm{H}_{8}\right.$ of quinoline), $8.12\left(\mathrm{~s}, 2 \mathrm{H}, \mathrm{H}_{4}\right.$ of quinoline $), 8.36-8.38\left(\mathrm{~d}, 2 \mathrm{H}, \mathrm{H}_{7-}\right.$ quinolin), 8.60-8.62(s, 2H, $\mathrm{H}_{5}$ of quinoline); Mass: $\mathrm{m} / \mathrm{z} 552.28\left(22.50, \mathrm{M}^{+}\right), 554\left(7.6, \mathrm{M}^{+2}\right)$, ${ }^{13}$ CNMR (DMSO-d6) $\delta: ~ 29.1, ~\left(\mathrm{CH}_{3}\right), 41.8\left(\mathrm{CH}_{2}\right.$-benzodiazepine), 64.3(C=N), 114.7, 119.1, 123.6, 124.3, 125.7, 125.9, 127.7, 127.9, 128.5, 129.7, 129.9, 130.2, 132.1, 132.6, 132.8, 135.2, 136, 131.3, 145.3, 145.2, 146.3, 152.6, 153.2, 163.8. Anal. Calc. For $\mathrm{C}_{28} \mathrm{H}_{18} \mathrm{Cl}_{4} \mathrm{~N}_{4}$ : C, 60.89; H, 3.29; N, 10.14. Found, C, 60.73; H, 2.98; N, 10.42 .

Table 1. Reaction time, yield and concentration of aliphatic acids for synthesis of newly synthesized[ 1,5$]$ - benzodiazepines 6a-f

\begin{tabular}{|c|c|c|c|c|c|c|c|c|c|}
\hline & \multirow{2}{*}{$\begin{array}{l}\text { Catalyt } \\
\text { Mol, \% }\end{array}$} & \multicolumn{8}{|c|}{ Reaction time / \% of yield } \\
\hline & & Malonic & Cinnamic & oxalic & Suucinic & formic & $\begin{array}{c}\text { Trichloro } \\
\text { acetic }\end{array}$ & tartaric & Chloroacetic \\
\hline \multirow[t]{4}{*}{$\mathbf{6 a}$} & 5 & $6 / 75$ & $6 / 75$ & $6 / 65$ & $6 / 70$ & $5 / 80$ & $5 / 82$ & $6 / 60$ & $5 / 85$ \\
\hline & 10 & $6 / 80$ & $6 / 82$ & $6 / 70$ & $6 / 75$ & $5 / 85$ & $5 / 83$ & $6 / 63$ & $5 / 92$ \\
\hline & 15 & $6 / 80$ & $6 / 82$ & $6 / 70$ & $6 / 75$ & $5 / 85$ & $5 / 85$ & $6 / 63$ & $5 / 92$ \\
\hline & 20 & $6 / 80$ & $6 / 82$ & $6 / 70$ & $6 / 75$ & $5 / 85$ & $5 / 85$ & $6 / 63$ & $5 / 92$ \\
\hline \multirow[t]{4}{*}{ 6b } & 5 & $6 / 77$ & $6 / 77$ & $6 / 68$ & $6 / 72$ & $5 / 82$ & $5 / 85$ & $6 / 62$ & $5 / 86$ \\
\hline & 10 & $6 / 87$ & $6 / 86$ & $6 / 74$ & $6 / 79$ & $5 / 89$ & $5 / 87$ & $6 / 63$ & $5 / 93$ \\
\hline & 15 & $6 / 87$ & $6 / 86$ & $6 / 74$ & $6 / 79$ & $5 / 89$ & $5 / 87$ & $6 / 63$ & $5 / 93$ \\
\hline & 20 & $6 / 87$ & $6 / 86$ & $6 / 74$ & $6 / 79$ & $5 / 89$ & $5 / 87$ & $6 / 63$ & $5 / 93$ \\
\hline \multirow[t]{4}{*}{ 6c } & 5 & $6 / 74$ & $6 / 71$ & $6 / 65$ & $6 / 68$ & $5 / 77$ & $5 / 75$ & $6 / 60$ & $5 / 86$ \\
\hline & 10 & $6 / 78$ & $6 / 75$ & $6 / 70$ & $6 / 74$ & $5 / 81$ & $5 / 80$ & $6 / 61$ & $5 / 95$ \\
\hline & 15 & $6 / 78$ & $6 / 75$ & $6 / 70$ & $6 / 74$ & $5 / 81$ & $5 / 80$ & $6 / 61$ & $5 / 95$ \\
\hline & 20 & $6 / 78$ & $6 / 75$ & $6 / 70$ & $6 / 74$ & $5 / 81$ & $5 / 80$ & $6 / 61$ & $5 / 95$ \\
\hline \multirow[t]{4}{*}{ 6d } & 5 & $6 / 60$ & $6 / 71$ & $6 / 65$ & $6 / 70$ & $5 / 80$ & 1582 & $6 / 60$ & $5 / 85$ \\
\hline & 10 & $6 / 61$ & $6 / 73$ & $6 / 67$ & $6 / 74$ & $5 / 85$ & $5 / 85$ & $6 / 63$ & $5 / 93$ \\
\hline & 15 & $6 / 61$ & $6 / 73$ & $6 / 67$ & $6 / 74$ & $5 / 85$ & $5 / 85$ & $6 / 63$ & $5 / 93$ \\
\hline & 20 & $6 / 61$ & $6 / 73$ & $6 / 67$ & $6 / 74$ & $5 / 85$ & $5 / 85$ & $6 / 63$ & $5 / 93$ \\
\hline \multirow[t]{4}{*}{$6 e$} & 5 & $6 / 62$ & $6 / 75$ & $6 / 65$ & $6 / 70$ & $5 / 80$ & $5 / 81$ & $6 / 62$ & $5 / 85$ \\
\hline & 10 & $6 / 64$ & $6 / 77$ & $6 / 66$ & $6 / 75$ & $5 / 85$ & $5 / 85$ & $6 / 65$ & $5 / 92$ \\
\hline & 15 & $6 / 64$ & $6 / 77$ & $6 / 66$ & $6 / 75$ & $5 / 85$ & $5 / 85$ & $6 / 65$ & $5 / 92$ \\
\hline & 20 & $6 / 64$ & $6 / 77$ & $6 / 66$ & $6 / 75$ & $5 / 85$ & $5 / 85$ & $6 / 65$ & $5 / 92$ \\
\hline \multirow[t]{4}{*}{ 6f } & 5 & $6 / 65$ & $6 / 72$ & $6 / 65$ & $6 / 70$ & $5 / 80$ & $5 / 82$ & $6 / 65$ & $5 / 85$ \\
\hline & 10 & $6 / 70$ & $6 / 75$ & $6 / 67$ & $6 / 74$ & $5 / 85$ & $5 / 87$ & $6 / 68$ & $5 / 94$ \\
\hline & 15 & $6 / 70$ & $6 / 75$ & $6 / 67$ & $6 / 74$ & $5 / 85$ & $5 / 87$ & $6 / 68$ & $5 / 94$ \\
\hline & 20 & $6 / 70$ & $6 / 75$ & $6 / 67$ & $6 / 74$ & $5 / 85$ & $5 / 87$ & $6 / 68$ & $5 / 94$ \\
\hline
\end{tabular}




\section{RESULT AND DISCUSSION}

First of all we study the catalytic efficiency of some aliphatic acids as malonic acid, cinnamic acid, oxalic acid, succinic acid, formic acid, trichloroacetic acid, tartaric acid, chloroacetic acid and we find in addition all the above acids has catalytic efficiency in progress of the condensation process in good yield were studied. Chloroacetic acid was found to give the best result for the easy, short time taking and high yield synthesis of new 1,5-benzodiazeines derivatives (Table 1). The catalyst quantity was optimized to $10 \mathrm{~mol} \%$ of chloroacetic acid and excellent results (95\% yields) were achieved.

\section{CONCLUSION}

In our work we study the catalytic activity of various available aliphatic acids and we found chloroacetic acid is efficient, inexpensive catalyst for one-pot synthesis of new 1,5-benzodiazepines (6a-f). This condition is highly productive procedure that make this work attractive for synthesis of 1,5-benzodiazepine.

\section{REFERENCE}

Ahmad S and Ali M, (2007): Iran. J. Chem .Eng., 26, 93-97.

Cyrous $\boldsymbol{O}$.Kangani, David E .Kelly,Billy W.Day, (2006): Tetrahedron letters , vol.47,35, 6289-92.

Di Braccio M, Grossi G C, Roma G, Vargiu L, Mura M and Marongiu M, (2001): Eur. J. Med. Chem., 36, 935-949.

Kamal M El-Gaml, (2012): One-Pot Synthesis And Antimicrobial Screening Of New [1,5]-Benzodiazepine Derivatives Catalyzed By Tbab, Al-Azh .j. ph. Sci, vol. $46,161-168$.

Kamal A, Shankaraiah N, Prabhakar S, Reddy C R, Markandeya N, Laxma K and Devaiah X, (2008): Bioorg. Med. Chem. Lett., 18, 2434-2439.

Konda S G, Shaikh B M, Chavan S A and Dawane B S, (2011): Chin Chem Lett., 22, 6568.

Kumar R and Joshi Y C, (2007): ARKIVOC, 13, 142-149.

Monir A S, Mohamad M I, Saber.E S B, Ashraf A A, Ashraf H B, Kamal M A El-Gaml, (2004): Bull. Pharm. Sci.-Assiut. Univ, vol.27, part2, 237-245

More $\boldsymbol{U}$ B, Kharat $\boldsymbol{R}$ S and Mahulikar P P, (2011): Asian J Chem., 23, 4311.

Neelima, Bhat B, Amiya P B, (1986): J. Het. Chem., 23, 295, 925-930.

Pasha M A and Jayashankara V P, (2006): J Pharm Toxicol., 6, 573-578.

Randall L O, (1974): Psychopharmacological Agents, Ed., Gordon M, New York, Academic Press, 3, 175-281.

Roma G, Grossi G C, Di Braccio M, Ghia M and Mattioli F, (1991): Eur J Med Chem., 26, 489.

Shi R X, Liu Y K and Xu Z Y, J. (2010): Zhejiang University, 11(2), 102-108.

Varala R, Enugala R and Adapa S R, (2007): J Braz Chem Soc., 18(2), 291-296.

Wright T.L., (1985): U.S.Pat.4,540,786 
تثييا لمشتقات ــ, 1 - بينزودايزبين حفزت بأحماض أليفاتية متنوعة

$$
\text { كمال محمد الجمل }
$$

قسم الكيمياء العضوية كلية الصيدلة (بنين) - جامعة الأزهر بالقاهرة

تم تشييد سلسلة جديدة من مشتقات او هـ بينزودايزبين بتكاثف الأورثوفينيلين ثنائى أمين مـع مشتقات ميثنيل

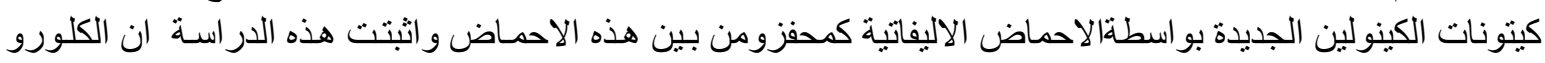

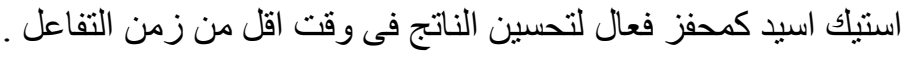

